\title{
Michael Cole
}

\section{Culture and Cognitive Science}

\section{Summary}

The purpose of this paper is to review the way in which cultural contributions to human nature have been treated within the field of cognitive science. I was initially motivated to write about this topic when invited to give a talk to a Cognitive Science department at a sister university in California a few years ago. My goal, on that occasion, was to convince my audience, none of whom were predisposed to considering culture an integral part of cognitive science, that they would indeed benefit from recognizing some affinities between the ideas of some of the founders of cognitive science and ideas about culture emanating from the Soviet (now Russian) cultural-historical school. My task in presenting this argument to the readers of Outlines is most likely the mirror image of that earlier effort. On the one hand, the ideas of the cultural-historical school are well known to this readership and you do not need to be lectured on the topic by an American whose knowledge of the topic is no greater than your own. At best, the ways in which I have appropriated those ideas and put them to work might provide an opportunity to reflect on the strange fate of ideas when they move between national traditions of thought. On the other hand, owing to a double twist of fate (after all, what was an American doing in Moscow in 1962 doing post-doctoral work in psychology) I was also present during the discussions leading to the founding of Cognitive Science in the early 1970's and subsequently became a member of the Cognitive Science Program at UCSD in the early 1980 's, arguably one of the pioneering efforts to institutionalize this new discipline.
My hope is that this unusual confluence of experiences, and the ideas that they have generated, will be of some use to those who see value in a dialogue between these different intellectual projects. With this goal in mind, I will begin by providing my own brief history of key ideas associated with the origins of cognitive science. My presentation will of necessity be highly selective - it is the relevance of the inclusion of culture in cognitive science that is my major focus.

I will then summarize some major milestones in the development of cognitive science at UCSD before turning to describe my own fusion of ideas from cultural-historical psychology and cognitive science as a kind of existence proof of the potential value of interdisciplinary dialogue.

\section{Culture and Cognitive Science "In the Beginning"}

Howard Gardner's "authorized biography" of cognitive science which treats its early origins and development up to approximately two decades ago can serve as a useful point of departure (Gardner 1985). Gardner begins his account with a series of scientific and technical advances that took place across the 1940's, 50 's, and 60's which set the stage for the new discipline. Among these were: 
1. The work of mathematicians such as Alan Turing and John von Neuman on computability and the feasibility of constructing computing machines that could work from programs stored in their own memories.

2. The work of Warren McCulloch and Walter Pitts showing that the operation of nerve cells and their connections to other nerve cells (neural networks) could be modeled in terms of logic.

3. Norbert Wiener's work on cybernetics in which he explicitly linked understanding of the human nervous system, electronic computers, and the importance of feedback from the environment.

4. The work of Shannon and Weaver on the development of information theory and George Miller's application of some of these ideas to the study of human memory in information processing terms.

5. Noam Chomsky's formalizations of grammatical competence.

6. A growing belief that stimulus-response versions of behaviorism had failed to produce on their promise, presaging the socalled cognitive revolution in psychology.

Responding to these and allied events, the Alfred Sloan Foundation sponsored a series of conferences bringing together representatives of the many disciplines implicated in Gardner's list of originators of cognitive science in the mid-1970's to explore the wisdom of providing support for a new, academic enterprise that synthesized these varied, and already interacting trends. The Foundation did indeed provide startup funding for some large programs in Cognitive Science, in effect launching the new discipline.

In the story as told by Gardner, the Foundation commissioned a "state of the art report" that summarized the ideas coming out of its series of conferences. The report declared the emergence of the field of cognitive science, explaining this new field in the following terms:
What has brought the field into existence is a com- mon research objective: to discover the represen- tational and computational capacities of the mind and their structural and functional representation in the brain (p. 36).

The report's authors represented the set of disciplines that had merged around this common objective as a "cognitive Hexagon" and attempted, through the use of dotted and solid lines to indicate the relationship among the constitutive disciplines (See Figure 1): Philosophy, Linguistics, Neuroscience, Artificial Intelligence, Psychology, and Anthropology.

This report was never published. According to Gardner, scholars representing every node of the hexagon felt under-represented, misrepresented, or otherwise in disagreement about the conceptual structure of the new discipline.

As a consequence of these divisions of opinion, combined with the firm resolve of Sloan to go ahead with its plans, a variety of differently constituted departments of cognitive science were created that represented different coalitions formed by sub-sets of the cognitive hexagon. In some places Artificial Intelligence (AI) came together with Psychology and Neuroscience; in others, Linguistics, Psychology, and AI, were combined, and so on. Common across all of these efforts was the centrality of modeling cognitive processes on digital computers.

Equally noticeable by its extreme rarity in the array of burgeoning cognitive science programs was the contribution of Anthropology, the discipline which held title to the concept of culture. Only in two major programs that I am aware of - the program put together at Berkeley (originally in collaboration with Stanford) and the program at UCSD - made the anthropological element of the cognitive hexagon a significant part of its mix. 


\section{Gardner's Hexagon}

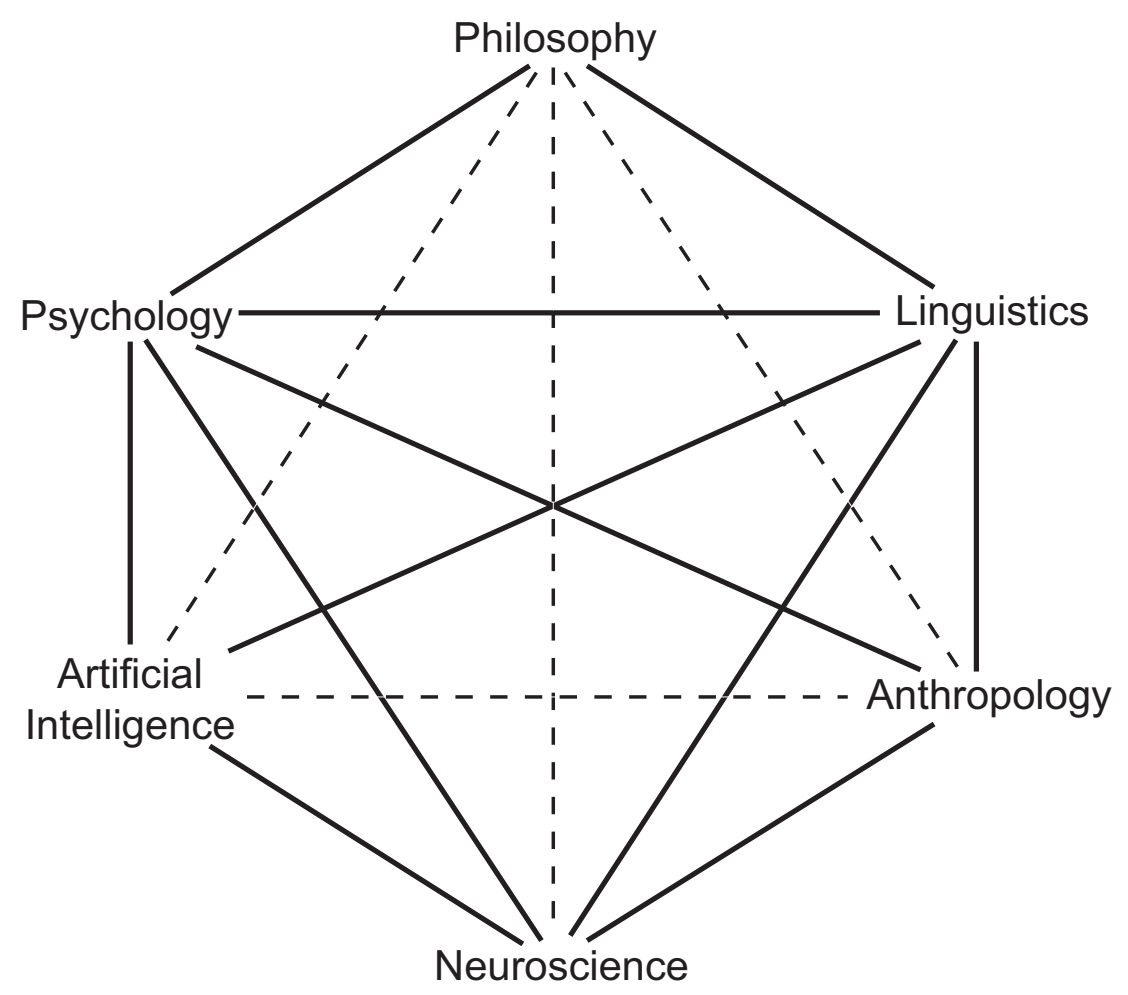

Connections among the Cognitive Sciences

Key: Unbroken lines = strong interdisciplinary ties

Broken lines $=$ weak interdisciplinary ties

Figure 1

Despite its failure to fulfill the ambitious goals of the Sloan Foundation's broad vision of the field, Gardner believed that the founders of cognitive science had made admirable progress toward their goal. The central achievement of this movement, as it appeared to Gardner in 1984, was to demonstrate the centrality of representation, or as he put it, "the clear demonstration of the validity of positing a level of mental representation: a set of constructs that can be invoked for the explanation of cognitive phenomena, ranging from visual perception to story comprehension (p. 383).

Writing only a few years later, Gardner noted two trends in the field which he linked to accomplishments and trends set by the early progenitors of cognitive science in the 1930-40's (Gardner 1987). The first was a vast increase in efforts to re-connect cognitive psychology with the neurosciences - the individual's mind with the individual's brain. The second trend was toward connectionist 
modeling, which highlights parallel processes and reduces the relative centrality of representation-as-internal-state. Gardner pointed to mixed systems employing combinations of the serial and parallel architectures as a promising way forward.

Curiously, in his retrospective look at the field, Gardner (1987) neglected to comment on another change - the increased influence of ideas about culture and cognitively-motivated ethnographic research as a significant, and challenging, sector within the Cognitive Science community. This seems an odd omission, because from remarks written in the earlier edition, we know that Gardner saw anthropology entering the field through work of myself, Jean Lave, Sylvia Scribner, and Ed Hutchins. Our approaches, he wrote, provide one way "that the anthropological community can have its cake and eat it too. ... by continuing the careful case studies that have been the lifeblood of the field, but to inform these studies with promising concepts or methods from cognitive science" (p. 256).

What Gardner could not see at the time, because the changes were taking place imperceptibly from individual scholars' perspectives, but what the advantage of hindsight affords us, is the realization that several interconnected changes were occurring that, taken cumulatively, were to bring about major new trends in the field. He saw the first serious challenge to the strong representationalist view in the work of Rumelhart, Norman, and McClelland on parallel distributed processing systems. But he did not see the cascading series of elaborations of these ideas that has ensued over the past decade nor could he discern the growing interest of contemporary anthropologists, psychologists, and others in the study of cognition as situated action occurring in context, or what some term, "embodied cognition." Each of these events appear to have brought the discipline to the point where the concepts of symbol manipulation on internal mental representation that
Gardner deemed the highest achievement of the discipline and the marginalization of culture and context would both undergo concerted attacks that makes it now possible to speak of a "new generation" of cognitive science that comes much closer to realizing the ideal of the cognitive hexagon than anyone anticipated even a decade ago.

I am not competent to survey all of the major changes that have occurred in cognitive science over the decade since Gardner wrote his highly informative description of the field and thoughts on its future. Rather, I want to focus specifically on the changing role of culture in contemporary cognitive science theories, and even more specifically on the line of thinking that grew up at UCSD. For anyone interested in thinking about how to build a cognitive science, perhaps my account can be useful, in particular if you are interested in making the study of culture in mind an intimate part of how you conceive your strategy. The experience of UCSD is also, I would argue, interesting in itself because of the unique contributions made by UCSD scholars to the study of culture as an intrinsically necessary part of cognitive science.

\section{CHIP: The Medium of Discussion}

I arrived at UCSD in the summer of 1978 after 9 years in the behavioral science group at the Rockefeller University. Trained several years earlier in mathematical learning theory by William Estes, my laboratory mixed experimental, comparative, cross-cultural studies with an increasingly ecologically-oriented approach in which I began to study cognition in ways that mixed experimental and ethnographic methods. This latter trend brought me together with George Miller, whose laboratory for the study of language acquisition served simultaneously as my laboratory for the study of cognitive task specification in non-laboratory settings. 
Table 1. Don Norman's (1980) 12 Issues for Cognitive Science

\begin{tabular}{|l|l|l|l|}
\hline Belief Systems & Emotion & Learning & Performance \\
\hline Consciousness & Interaction & Memory & Skill \\
\hline Development & Language & Perception & Thought \\
\hline
\end{tabular}

By virtue of proximity, I had been present for many of the Sloan Foundation meetings. It was my view that anthropologists were going to have a difficult time getting their voices heard, except in so far as the discipline restricted itself to a particular form of "cognitive anthropology" which sought to specify the systems of concepts that represent the content of cultural knowledge. While I found this work interesting and important, and I had used several of the associated methods in my own prior work, I was bothered by the absence of a different side of cognitive anthropology, one in which the socio-culturally organized environment in all of its materiality and sensitivity to locally constructed contexts plays an important role in how cognition works.

When I came to UCSD in 1978, becoming involved in cognitive science was not one of my imagined goals. But I was very interested in the work of several UCSD faculty in psychology, anthropology, and sociology, which, I thought, provided a really interesting intellectual environment for working out a way to see culture and context co-equal constituents with representation and information processing in how one conceives of the function of human psychological processes.

During the 1978-1979 academic year, I organized a seminar that included Aaron Cicourel, Roy D'Andrade, George and Jean Mandler, Don Norman, Bud Mehan, and Dave Rumelhart to explore ways to bring notions of cognition and notions of cultural context together within a single framework. Shortly thereafter, Don Norman, who played an essen- tial role in institutionalizing Cognitive Science, created a cognitive science program which was the breeding ground for Parallel Distributed Processing models and the development of connectionism. All of the members of our cognition and context seminar participated.

The first products of these interactions (which Don characterized as "fierce and useful" (Norman 1980), p. 31) appeared in print in 1980 when Don published "Twelve issues for Cognitive Science" in the journal, Cognitive Science, which became the official organ of the new Cognitive Science Society. Don's twelve issues are worth listing for what they tell us about how culture did and did not enter into his thinking about the discipline he envisioned (See Table 1).

The most conspicuous way that culture enters this list is in the first entry, belief systems. The late 1970's were a time when both cognitive psychologists and cognitive anthropologists were interested in such concepts as schema, script, and story grammars, conceived of as structured internal representations. In this form, culture fit comfortably into the field as Don then conceived of it. The important work of Roy D'Andrade on cultural models as cognitive schemas provides an outstanding example of this approach (D'Andrade and Posner, 1989). Assuming that culture entered cognition in the form of cultural schemas, Don was able to picture his overall conception of the cognitive system in the following diagram (Figure 2).

I was not happy with the characterization of the basic constituents of the model Don presented, and the ensuing discussions made 


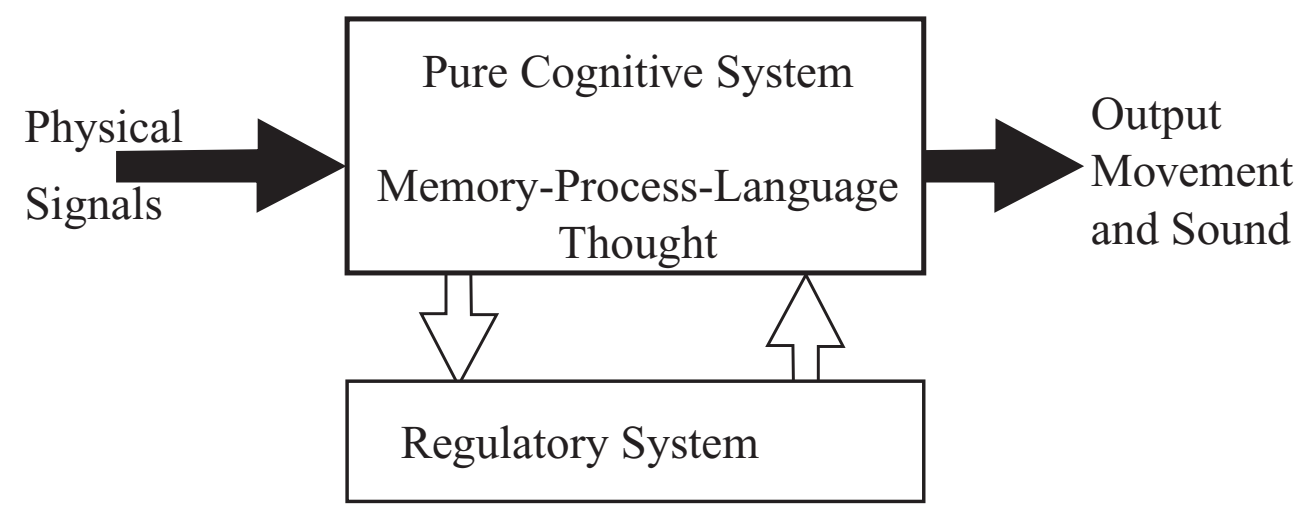

Figure 2

enough of an impression on him to motivate him to tack a discussion of "The environmental system and cultural knowledge" onto his twelve issues. The core of my argument was that he needed to consider the Environmental System (ES), consisting of physical and social parts, as an equal partner in giving shape to the super-system comprised of RS and PCS (The regulatory and so-called pure cognitive systems).

This re-conceptualization, I argued, would enable Don to make a much stronger case for the relevance of evolutionary neuroscience, developmental psychology, and cognitive anthropology. Why? Because the mature system that encompasses RS and PCS must develop through a series of interactions between the RS and the ES. The PCS should be seen as an evolving adaptation. Where does culture enter? At some point it becomes part of the ES, one with an external source of memory over generations to supplement the "memory" built in by evolution.

“... What culturally organized knowledge does is to carry a lot of information for us ... so a lot of the processes that experiments require to be done in the head can be, and is, short-circuited in real life ... One issue is how to describe cognition as an interaction between the head and the world where some of the thought power resides in each locus" (Quoted in Norman, 1980, p. 28).
Several years later, Don, influenced primarily, I believe, by Ed Hutchins, came more and more to take seriously the role of the culturally organized environment of cognition. In 1988 he published The Psychology of Everyday Things, and in 1993, Things That Make Us Smart. In this and corollary work, Don and Ed Hutchins promoted the idea of "distributed cognition," an idea to which I will return because it provides one important way of articulating a view of cognitive science in which culture is seen to play a central role. A key concept around which our discussions revolved, and one which provides clear links back to the classical origins of cognitive science, in addition to providing a natural way to include culture in cognitive science, was the idea of an artifact.

\section{Artifacts in The Science of the Artificial}

My own approach to thinking about artifacts owes its origins to that branch of scholarship referred to as cultural-historical psychology (Chaiklin and Hedegaard, 1999) or as I and my colleagues have come to conceive of it as cultural-historical activity theory (Engeström, Miettinen et al. 1999). It is one of a broad family of theories that take the mediation of human action through artifacts to be the central mo- 
ment in the constitution of human mind. My entry into this way of thinking starts from the Russian tradition initiated by Vygotsky, Luria, Leontiev, and their colleagues, and the American tradition represented by John Dewey and George Herbert Mead. It incorporates the work of Bakhtin, a literary/cultural theorist, Ilyenkov, a philosopher, Kenneth Burke, a philosopher/literary theorist, and Gregory Bateson, ecologist of the mind, as well as related traditions from Germany, Scandinavia, England, France, and other parts of Europe.

According to this view, an artifact is an aspect of the material world that has been modified over the history of its incorporation in goal directed human action. By virtue of the changes wrought in the process of their creation and use, artifacts are simultaneously ideal (conceptual) and material. Artifacts are material objects, created in the process of goal directed human actions. They are ideal in that their material form has been shaped by their participation in the interactions of which they were previously a part and which they mediate in the present.

I do not think it an exaggeration to say that from this perspective, human thinking is artificial. Human thought can be conceived as the deformation of "naturally occurring" behavior, that is, behavior not mediated by culture. The acquisition of culture and the acquisition of thought are part of a single process, both in the normal thinking of adults and in the process of hominization. However, this assumption only sets the stage for addressing the relationship between cultural-historical psychology and the scientific enterprise called Cognitive Science.

\section{The Artifact in Classical AI}

It is instructive to compare this conception of artifact-mediated mind with the conception of artifacts found in one of the undisputed progenitors of cognitive science, the study of arti- ficial intelligence. To psychologists and the lay public alike, the notion of artificial intelligence conjures up the image of a digital computer, programmed to implement a formal symbolmanipulating system that solves recognizable problems. In many interpretations, physical symbol systems embodied in computers are thought of as analogous to, if not direct models of, the operation of the human brain (Gardner, 1985; Newell, Rosenbloom et al. 1989). A difficulty with the classical AI view from the perspective of a cultural-historical approach to mind, as many have pointed out, is that it adheres to a strong Cartesian separation between what is outside and what is inside the mind. (So, for example, Alan Newell, Rosenbloom, and Laird, 1989, p. 107, could write: "Symbol systems are an interior milieu, protected from the external world, in which information processing in the service of the organism can proceed"). This strong separation between outside and inside provides an important difference between classical AI and the position that Don developed on the one hand, and my own version of a cultural-historical approach.

\section{Simon's definition of the artificial}

In his influential monograph, The Sciences of the Artificial (1981) Herbert Simon provided a canonical AI account of the study of human thought within the discipline of artificial intelligence. He took as his central concern the family of artifacts called physical symbol systems. "Symbol systems," he wrote, "are almost the quintessential artifacts, for adaptivity to the environment is their whole raison d'etre (Simon 1981, p. 27).

Simon offers four criteria that distinguish the artificial from the natural:

1. Artifactual things are synthesized (though not always or usefully with full forethought) by man.

2. Artificial things may imitate appearances 
in natural things while lacking, in one or many respects, the reality of the latter.

3. Artificial things can be characterized in terms of functions, goals, adaptation

4. Artificial things are often discussed, particularly when they are being designed, in terms of imperatives as well as descriptives (1981, p. 8).

Simon's equation of the artifactual with things synthetic reveals a deep affinity between these two concepts. To be synthetic, according to Webster's dictionary, means to be "produced by chemical synthesis, rather than of natural origin; not real or genuine; artificial." So in dealing with creatures whose minds are made through artifacts, we are dealing with hybrids, part natural, part cultural-historical.

Points 2-4 also provide interesting points of contact between Simon's notion of artificial, and the notion of artifact that comes out of cultural-historical theory. Artifacts are, in some respects, models. Their structures carry within them, so to speak, a "theory" of both the human who is using them and the range of environmental circumstances in which they will be normatively used. Every axe and hammer, for example, embodies such a theory in its length, its shape, its size, its weight as a synthesized ensemble that satisfices the constraints of the human user and the task at hand. At the same time artifacts are transformers, enabling the metamorphoses of what we refer to as external into internal and vice versa. Because they enter intimately into human goal directed action, there is a functional aspect to all artifact-mediated action. And for the same reasons artifacts embody values (oughts, shoulds, and musts); in this sense all culturally mediated action is, at least implicitly, moral action.

I take Simon's characterization of artifacts to be compatible with a cultural-historical approach to human psychological functioning so long as we realize that none of his criteria justifies, let alone requires, the reduction of artificial intelligence to an inside-the-head, cutoff-from-the-world, physical symbol system. Nor need the criteria be embodied in computers. As Don Norman and Ed Hutchins have amply demonstrated, many systems of activity, such as those engaged in by 747 pilots, fill Simon's criteria quite adequately.

\section{Cognitive Artifacts}

In 1990, Don wrote a paper entitled "Cognitive artifacts." His goal, as he expressed it, was to emphasize the information processing role played by physical artifacts upon the cognition of the individual - hence the term, "cognitive artifact." "Here, I will not be concerned with how they are invented, acquired, or transmitted across individuals or generations. The goal is to integrate artifacts into the existing theory of human cognition (Norman 1991, p. 2).

This passage, and the ensuing discussion, are important because they pinpoint two key ways in which Don's approach differed from my own, despite a growing area of convergence in our ways of thinking. First, I call attention to the idea that cognitive artifacts "play a role upon the cognition of the individual." Here we see the persistence of a deep intuition that thought is an autonomous human activity and that artifacts are somehow external to human thought, acting upon it, rather than participating in it and constituting it. This intuition is inscribed, as well, in the diagrams that Don provided to contrast two views of artifacts, what he called the personal and the system views (see Figures 3 and 4).

As indicated in the passage I have quoted, as well as the figure legends to the two figures, there is no intrinsic relationship between thought and artifact. Rather, artifacts are placed squarely "outside" the cognitive system, acting upon it. From the former view, artifacts enhance cognition, from the latter view they simplify the task, but the basic schism between task and cognition remains unaffected. Cogni- 

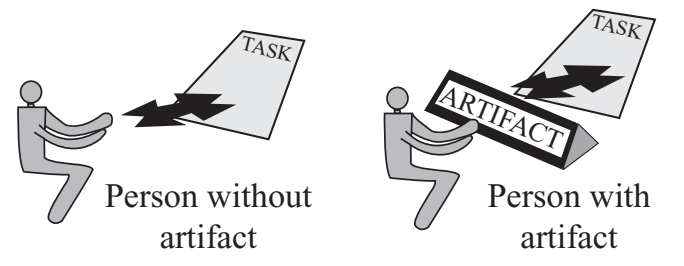

Figure 3 and 4

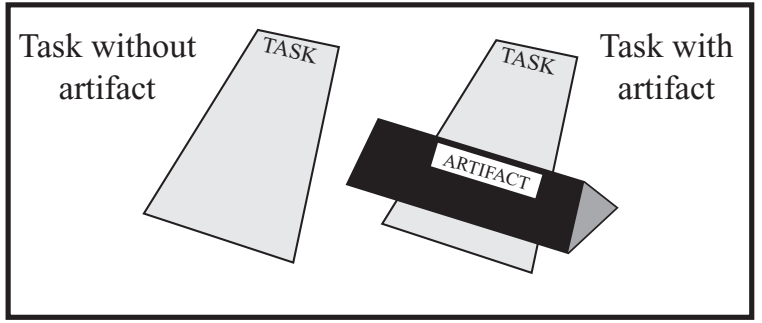

tion is a process occurring in individual heads and the basic stimulus-response paradigm remains in place. Ed Hutchins, who worked closely with Don during this period, arrived at a different way of looking at artifacts that I believe to be a variant of my own approach. On the basis of extensive research on the work of airplane pilots, Ed began to evoke the notion of a "functional system", which he adapted from the writings of Alexander Luria. Instead of viewing individuals, artifacts, and tasks as independent entities, Ed argued that the process of human thinking entails bringing different sub-systems into coordination with each other. As a consequence, the basic processes of cognition are organized differently by different task conception:

The really important insight here is that some of the systems that are brought into coordination in thinking may be outside the usually conceived boundaries of the person. Such a system of complex (and dynamic) coordination of other systems is a "functional system". Different functional systems are set up in the performance of different tasks, and, since the functional system may include coordination with symbolic systems outside the person, a change in the symbolic resources may lead to a reconfiguration of thinking (Hutchins, 2000, p. 4).
Within this framework, cognitive artifacts do not simply act on the individual, they are constitutive of the information processing mechanisms that cognitive science takes as its object of study. Elaborating on this view in a recent article on cognitive artifacts, Hutchins makes the incestuous connections between cognition, cognitive artifacts, tasks, and the broader socio-cultural environment explicit:

Cognitive artifacts are always embedded in larger
socio-cultural systems that organize the practices
in which they are used. The utility of a cognitive
artifact depends upon other processes that create the
conditions and exploit the consequences of its use.
In culturally elaborated activities, partial solutions
are often crystallized in practices, in knowledge,
and in social arrangements (Hutchins 1999, p. 4).

Ed brings two sources of data to bear upon this alternative, "distributed cognition" view which makes artifacts constitutive of thought, not clearly parsable into internal and external influences. First, there are careful "cognitive ethnographies" of a variety of work-related activities such as navigating a large naval vessel or flying an airplane. Second, there are implementations of connectionist models ("individuals") that are placed in interaction with each other, and which, 
in Ed's most recent work, include interactions with the products of past interactions (artifacts), thereby instantiating in computer simulations the process of cultural mediation as intrinsic to human cognitive processes. An important idea to come from this latter work is the possibility that there may be jointly achieved, interpsychological functions which are sufficiently complicated that they never become independently realizable individual psychological functions, but can only be achieved as joint, mediated, activity in context. Needless to say, this possibility severely undermines reliance on the individual human mind, let alone the individual human brain, as the locus of cognition.

\section{From Cognitive Artifacts to the Fifth Dimension}

I believe that the path of my own work is consistent with the distributed cognition tradition. However, it explores another part of the spectrum of possible ways to investigate the mediation of human activity by "intelligent devices" into which enormous reserves of cultural understanding have been forged and it deals directly with the issue of development that was included in Don's twelve issues, but thereafter more or less ignored. I focus on child development and in particular on the period of middle childhood. Further, within that age-defined domain, I study development outside of the school, in marginal community organizations such as youth clubs, church groups, and afterschool extended-day educational programs.

My method is also different. When Ed Hutchins studies an existing functional system of activity such as an airplane cockpit, he does not pretend to be an invisible participant, and in Ed's case, he has "gone native" to be certified as a pilot so that he can learn about the system from the inside out. However, in most cases, participant observers are not responsible for the existence of the system they study. By virtue of the fact that it is a "real world" system they are investigating (an important virtue, because it displays the utility/believability of the theory), the activity they study would exist if they were not there to observe it; the ongoing activity of the observer does not require their participation.

By contrast, my students, colleagues, and I literally create the systems of activity that are the focus of our research. We are participant observers in a quite unique way. In this, I believe we are invoking an idea that has been common to both the cultural-historical tradition and to the study of artificial intelligence: you can best understand something you have made. However, we do not embody this principle by writing computer models of parallel distributed processing models that incorporate a mixture of natural and cultural processes or by conducting ethnographic observations of complex work settings in which computer-based cognitive artifacts are treated as elements in a functional system of artificial intelligence. Instead, we design systems of activity which embody principles of cultural-historical psychology and test our theories by their ability to create the forms of transformation that the theory takes as its central processes.

My approach differs from that of others currently working on the role of culture in mind via the study of artifact-mediated activity in another respect. Recall Don Norman's caveat that he was not engaged in the study of how artifacts are created, acquired or transmitted. From the perspective of a cultural-historical approach to cognition, this restriction has serious, negative, consequences. If, as Ed Hutchin's argues, cognition is a process of the propagation of structure across media within complex, dynamic, functional systems, then knowledge of the processes of transformation over time is vital to understanding how artifacts enter into the process of human thought. This process of transformation cannot be focused entirely on artifacts themselves. Rather, it must also lay 
bare for analysis the way in which an object/ activity and its context develop as part of a single process of change.

I refer to the activity systems I design as tertiary artifacts, following Marx Wartofsky (Wartofsky, 1973). Wartofsky identified as tertiary a special class of artifacts "... which can come to constitute a relatively autonomous 'world', in which the rules, conventions and outcomes no longer appear directly practical, or which, indeed, seem to constitute an arena of non-practical, or 'free' play or game activity" (p. 208). Engagement with such imagined worlds, he suggests, can come to color the way we see the "actual" world, providing a tool for changing current praxis. In modern psychological jargon, modes of behavior acquired when interacting with tertiary artifacts can transfer beyond the immediate contexts of their use. Wartofsky applies his conception of tertiary artifacts to works of art and processes of perception; I want to generalize his conception to include specially designed systems of activities for children. I call the systems we design, implement, and seek to sustain, "5th Dimensions."

The Fifth Dimension was designed to be an educational activity system that offers school aged children a specially designed environment in which to explore a variety of off-the-shelf computer games and game-like educational activities during the after school hours (at present, it is also implemented as part of the curriculum in some schools). This activity is participated in by undergraduates from a college or university. The computer games are a part of a make-believe play world that includes non-computer games like origami, chess, and mancala (the West African board game) and a variety of other artifacts. "Task cards" or "adventure guides" written by project staff members for each game are designed to help participants (both children and undergraduate students) orient to the game, to form goals, and to chart progress toward becoming an expert. The task cards provide a variety of requirements to externalize, reflect upon and criticize information, to write to someone, to look up information in an encyclopedia, and to teach someone else what one has learned, in addition to the intellectual tasks written into the software or game activity itself.

As a means of distributing the children's and undergraduates' use of the various games the Fifth Dimension contains a table-top or wall chart "maze" which permits distribution of activities in a flexible manner. This distribution device consists of some 20 "rooms". Each room provides access to two or more games, and the children may choose which games to play as they enter each room.

There is an electronic entity (a wizard/ wizardess, Maga, Golem, Proteo - depending upon the locally constructed mythology) who is said to live in the Internet. The entity writes to (and sometimes chats with) the children and undergraduates via the Internet. In the mythology of the Fifth Dimension, the wizard/ess acts as the participants' patron, provider of games, mediator of disputes, and the source of computer glitches and other misfortunes.

Because it is located in a community institution, the Fifth Dimension activities require the presence of a local "site coordinator" who greets the participants as they arrive and supervises the flow of activity in the room. The site coordinator is trained to recognize and support the pedagogical ideals and curricular practices that mark the Fifth Dimension as "different"a different way for kids to use computers, a different way of playing with other children, and a different way for adults to interact with children.

The presence of university and college students is a major draw for the children. The participating college students are enrolled in a course focused on fieldwork in a community setting. At UC San Diego, an institution that emphasizes research, the university course associated with student participation is an 
intensive, 6 unit class that emphasizes deep understanding of basic developmental principles, the use of new information technologies for organizing learning, and writing fieldnotes and research papers. The undergraduates write papers about the development of individual children, the educative value of different games, differences in the ways that boys and girls participate in the play world, variations in language use and site culture, and other topics that bring regular course work and field observations together. Different arrangements for undergraduate courses exist at each of the sites, depending upon local needs (see www.uclinks.org for descriptions of currently existing sites and research foci).

By virtue of their socio-ecological location, children cannot be compelled to attend a 5thDimension, they must come voluntarily, choosing this over other potential activities. As a consequence, a design feature common to all 5thDimensions is that they mix play and academically oriented activities. More than a decade of research designing, implementing, and seeking to sustain 5thDimensions has strongly reinforced my view that they can usefully be interpreted as systems of artificial intelligence, saturated with cognitive artifacts, in interaction with which children and adults can behave more intelligently (adaptively) in a wide variety of circumstances.

With respect to the child participants, time spent inhabiting the 5 thDimension has been demonstrated to produce increased performance on a variety of standard and specially designed problem solving and information processing tasks as well as increased social-interactional skills (this evidence is summarized in many papers and monographs obtainable through www.uclinks.org). For undergraduate students who participate in the 5thDimension, the experience brings about deeper conceptual understanding of the principles of learning and development as well as increased self confi- dence in dealing with children and technology. For the researchers, the 5thDimension provides a unique medium for understanding ontogeny as the emergent product of microgenesis and cultural-historical change in patterned cultural practices. We have been able to document the processes of artifact-mediated interaction that give rise to these consequences, providing evidence for the underlying theory and design principles. Comparative analysis of 5thDimensions in different institutional settings has clearly shown that although they start from a common set of elements and normative rules, each of the systems we "plant" in a new institutional setting becomes a unique synthesis of the initial set of artifacts and its specific cultural-historical location.

\section{Concluding Remarks}

In a brief article such as this, which seeks to cover a lot of ground, it is impossible to do justice to the all of the topics I have raised, many of which could have taken up the allotted space by themselves. My goal has been to suggest that the topic of culture as conceived of by cultural-historical approaches and in cognitive science is worthy of consideration in thinking about the development of both disciplines. If the general position I have put forth is correct, a cognitive science that proceeds without taking the human need and ability to mediate thought through artifacts, including artificially designed systems of activity, will prove limited in both theory and practice. As Andy Clark notes in his recent book, Being There, evidence concerning the intimate role of the environment in thought processes implies that classical AI "bundles into the machine a set of operational capacities which in real life emerge only from the interactions between machine (brain) and world (Clark 1997, p. 64)". Put even more bluntly, Ed Hutchins argues that an a-cultural cognitive science errs in "mistaking 
the properties of the sociocultural system for the properties of the person" (p. 366).

On the positive side, a cognitive science which does include artifact mediation in cultural historical context as part of its fundamental toolkit makes excellent contact with current attempts to understand human thought as the embodied activity of active agents, embedded in environments which are also active, embodied agents.

\section{References}

Chaiklin, S. and M. Hedegaard, Eds. (1999). Activity theory and social practice: culturalhistorical approaches. Aarhus, Aarhus University Press.

Clark, A. (1997). Being there. Cambridge, MA, MIT Press.

D’Andrade, R. G. and M. I. Posner (1989). Cultural cognition. Foundations of cognitive science. M. Posner. Cambridge, MA, MIT Press.: 795-830.

Engeström, Y., R. Miettinen, et al., Eds. (1999). Perspectives on activity theory. New York, Cambridge University Press.
Gardner, H. (1985). The mind's new science: A history of the cognitive revolution. New York, Basic Books.

Gardner, H. (1987). The mind's new science: a history of the cognitive revolution. Second Edition. New York, Basic Books.

Hutchins, E. (1999). Cognitive artifacts. The MIT encyclopedia of the cognitive sciences. R. A. Wilson and F. C. Keil. Cambridge, MA., MIT Press: 126-127.

Hutchins, E. (2000). The appropriation of cultural structure by cognitive processes. American Anthropological Association, San Francisco.

Newell, A., P. S. Rosenbloom, et al. (1989). Symbolic architectures for cognition. Foundations of cognitive science. M. Posner. Cambridge, MA, MIT Press.

Norman, D. A. (1980). "Twelve issues for cognitive science." Cognitive Science 4(1): 1-32.

Norman, D. A. (1991). Cognitive artifacts. Designing interaction. J. M. Caroll. New York, Cambridge University Press: 17-38.

Simon, H. (1981). Sciences of the artificial. Cambridge, MA., MIT Press.

Wartofsky, M. (1973). Models. Dordrecht: D., Reidel. 